\section{REFERENCES}

1 Marthan R, Castaing Y, Manier G, et al. Gas exchange alterations in patients with chronic obstructive lung disease. Chest 1985; 87: 470-475.

2 Wagner PD, Dantzker DR, Dueck R, et al. Ventilation-perfusion inequality in chronic obstructive pulmonary disease. J Clin Invest 1977; 59: 203-216.

3 Hagen PT, Scholz DG, Edwards WD. Incidence and size of patent foramen ovale during the first 10 decades of life: an autopsy study of 965 normal hearts. Mayo Clin Proc 1984; 59: 17-20.

4 Herve P, Petitpretz P, Simonneau G, et al. The mechanisms of abnormal gas exchange in acute massive pulmonary embolism. Am Rev Respir Dis 1983; 128: 1101-1102.

5 Oudiz RJ, Midde R, Hovenesyan A, et al. Usefulness of right-to-left shunting and poor exercise gas exchange for predicting prognosis in patients with pulmonary arterial hypertension. Am J Cardiol 2010; 105: 1186-1191.
6 Marshall C, Marshall B. Site and sensitivity for stimulation of hypoxic pulmonary vasoconstriction. J Appl Physiol 1983; 55: 711-716.

7 Jardin F, Delorme G, Hardy A, et al. Reevaluation of hemodynamic consequences of positive pressure ventilation: emphasis on cyclic right ventricular afterloading by mechanical lung inflation. Anesthesiology 1990; 72: 966-970.

8 Barbera JA, Roger N, Roca J, et al. Worsening of pulmonary gas exchange with nitric oxide inhalation in chronic obstructive pulmonary disease. Lancet 1996; 347: 436-440.

9 Blanco I, Gimeno E, Munoz PA, et al. Hemodynamic and gas exchange effects of sildenafil in patients with chronic obstructive pulmonary disease and pulmonary hypertension. Am J Respir Crit Care Med 2010; 181: 270-278.

10 Rietema H, Holverda S, Bogaard HJ, et al. Sildenafil treatment in COPD does not affect stroke volume or exercise capacity. Eur Respir J 2008; 31: 759-764.

\title{
Three colleagues with sarcoidosis?
}

\section{To the Editors:}

Bilateral pulmonary nodules are a relatively common finding in thoracic radiology and hence are an important problem that chest physicians often face. Aetiology can be diverse, ranging from neoplastic conditions to infectious lesions. An accurate and prompt diagnosis is needed in order to target the most appropriate and least harmful treatment. We report the case of a 49-yr-old, Caucasian, otherwise healthy male patient with bilateral lung nodules and an unusual diagnosis.

The patient had an unremarkable history and was referred due to asymptomatic bilateral lung nodules. He had stopped smoking 6 months earlier and had a normal chest radiograph a year before referral, taken for follow-up of benign colon polyps. At the time of presentation he was not on any medication.

The patient was an engineer working in a Belgian high-tech facility. His job mainly consisted of office work with occasional visits to production sites where coating of medical material was performed. When visiting these sites, he did not systematically wear a protective mask. The problems occurred after a business trip to the USA where he met with three colleagues. He first visited his general practitioner with a flu-like syndrome. Other symptoms included a productive cough, night sweats and loss of weight (5 kg in 2 weeks). He was treated symptomatically with paracetamol. Two of his colleagues developed the same complaints. One never visited a doctor but the other did and was diagnosed with sarcoidosis based on the finding of non-necrotising granulomatous lesions in transbronchial biopsies. He was put on steroids and his condition improved. Oddly, the third colleague who did not visit the doctor had an existing diagnosis of sarcoidosis but remained asymptomatic.

Due to persistent cough and low-grade fever, our patient was subsequently referred to a pneumologist. Biochemistry revealed an inflammatory syndrome (C-reactive protein $4.6 \mathrm{mg} \cdot \mathrm{dL}^{-1}$, sedimentation rate $28 \mathrm{~mm} \cdot \mathrm{h}^{-1}$ ) and normal tumour markers, angiotensin-converting enzyme (ACE) levels and anti-nuclear factor (ANF). Mycoplasma pneumoniae serology was compatible with a recent infection. Computed tomography (CT) of the chest showed bilateral lung nodules, some with cavitation, in a subpleural and more central distribution. Extensive mediastinal lymphadenopathy was also present. Bronchoscopy revealed a normal bronchial tree. Bronchoalveolar lavage was neutrophilic $(60 \%)$ with no bacterial pathogens and a normal cytology. Pulmonary function tests were normal (vital capacity $4.9 \mathrm{~L}$, forced expiratory volume in $1 \mathrm{~s}$ (FEV1) 4.4 L, FEV1/forced vital capacity (FVC) $88 \%$, total lung capacity $9.2 \mathrm{~L}$, diffusion capacity $99 \%$ ). Atypical pneumonia was diagnosed and the patient was treated with doxycycline $200 \mathrm{mg}$ daily for 10 days.

2 months after initial presentation the symptoms disappeared and biochemistry normalised. The lung nodules persisted but the cavitations and adenopathy regressed. The patient was then referred to our centre for a second opinion. At that time he was completely asymptomatic and the initial weight lost had been regained. Our differential diagnosis included chronic berylliosis, sarcoidosis and, less likely, malignancy and opportunistic infections. A lymphocyte transformation test (LTT) performed for berylliosis was negative. Bronchoscopy with lavage, brushing and biopsies was repeated and showed a normal cell count and differentiation, a CD4/CD8 ratio of 3.8 and no bacterial pathogens on cultures. Bronchial brushing and biopsies were normal. HIV test was negative.

5 months after his business trip the patient remained asymptomatic. No further change of the radiological picture was noted. Exposure to beryllium seemed very unlikely since the LTT was negative. Given the uncertainty of the diagnosis, a lung biopsy was performed. This revealed necrotising granulomas with a positive Grocott methenamine silver (GMS) stain (fig. 1). A serological test for Histoplasma capsulatum antibodies confirmed 


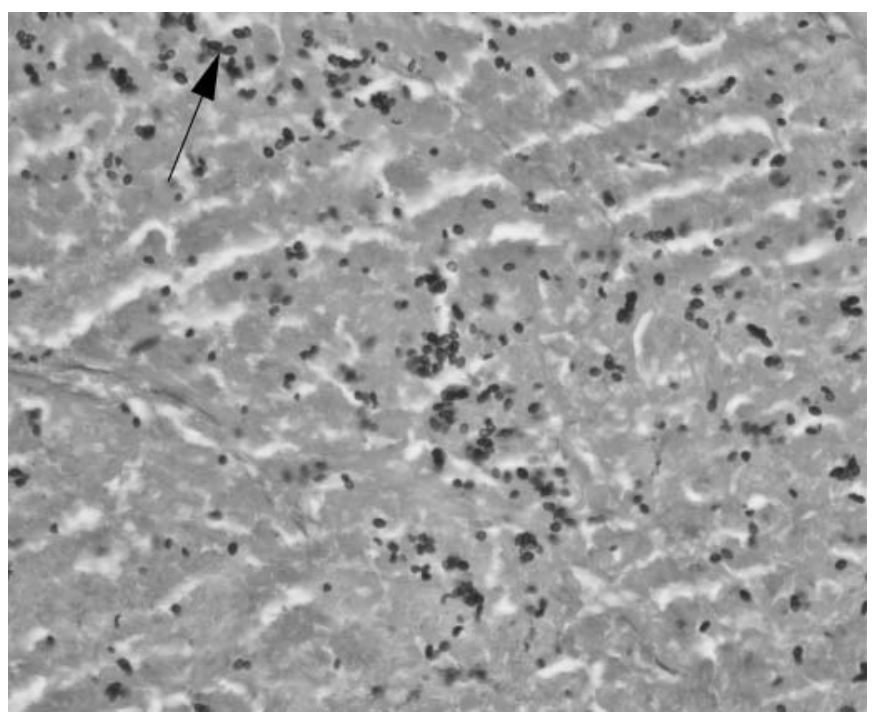

FIGURE 1. Necrotising granuloma from lung biopsy specimen. Small ovoid yeasts, some with narrow-based budding (arrow), representing Histoplasma capsulatum in a background of necrosis. Grocott methenamine silver stain. Original magnification $\times 200$.

pulmonary histoplasmosis. Our patient did not receive any specific treatment and remained clinically stable 6 months after initial presentation. Intriguingly, retrospective examination of the histopathological samples showed the presence of the same micro-organism in our patient's colleague who was diagnosed with sarcoidosis and treated with steroids. His clinical improvement seemed unexpected as steroids are known to depress the cellular immunity, which would facilitate the infection with $H$. capsulatum [1]. We did not ask to review the transbronchial biopsies of the third colleague as his diagnosis of sarcoidosis was prior to the business trip and he remained asymptomatic thereafter.

Granulomas are amongst the most common pathological findings in pulmonary medicine; yet diagnosis often remains challenging due to incomplete clinical data and the difficulty of interpreting some histological features. Granulomas are compact aggregates of histiocytes (macrophages). They may also contain necrosis (necrotising granuloma), lymphocytes, plasma cells or multinucleated giant cells [2]. Infections are the first cause of granulomatous lung disease, with mycobacteria and fungi as the two most common infectious agents. Sarcoidosis is a primary non-infectious cause, but other causes include chronic berrylium disease, hypersensitivity pneumonitis and, less frequenly, hot tub lung and Wegener's granulomatosis [2].

H. capsulatum is a dimorphic fungus existing as a mould in the soil and as a yeast at body temperature [1,3]. Soil containing bat or bird droppings is the reservoir. The fungus is endemic to certain regions such as North and Central America, but also to Africa, Southern Europe and South-Eastern Asia. Exposure in these locations is extremely frequent but symptomatic infection is less common and depends on the balance between cellmediated immunity and infectious burden. In an immunocompetent host, a large inoculum is needed to cause the disease, while immunocompromised patients are at risk of developing it with a much smaller inoculum. Contamination occurs with inhalation of the microconidia that reach the alveoli and infect the macrophages before spreading via the reticuloendothelial system $[1,3]$.

Clinical pictures can be acute or chronic, local or disseminated and possibly complicated. The acute pulmonary forms are a mostly mild pneumonia mimicking an atypical pneumonia and are thus often treated as such, as in our patient. Severe forms also exist and can lead to acute respiratory distress syndrome (ARDS). Chronic cavitary pulmonary histoplasmosis occurs generally in patients with emphysema, resembling a reactivation of tuberculosis. Complications of these pulmonary forms include granulomatous mediastinitis, fibrosing mediastinitis and pericarditis, but these are less common. Disseminated histoplasmosis can be either acute, leading to a sepsis-like illness, or chronic. Immunocompromised patients develop acute illness. Chronic disseminated histoplasmosis is seen in older, mostly male patients with underlying emphysema but normal immunity [1].

Diagnosis is via careful review of patient history and can be confirmed using various tests. The test used depends on the clinical syndrome [1, 2, 4]. For chronic histoplasmosis, serology is the test of choice. Complement fixation and immunodiffusion techniques can both be used. Antibody production takes 48 weeks, however, and repeating the test might be necessary in case of strong clinical suspicion and initial negative serology. False-positive serology can be seen in lymphoma, tuberculosis, sarcoidosis and other fungal infections. A persistent lowpositive titre can also be noted years after exposure and therefore does not always correspond to active infection [1]. Culture of respiratory specimen is also indicated in chronic pulmonary forms but pathogen growth may take weeks. In acute and particularly disseminated forms, antigen detection is rapid. Urinary testing has the best sensitivity. PCR techniques are not yet reliable. Histopathology can be divided into three main types [2]. The first is an intra-alveolar lymphohistiocytic infiltrate with small granulomas and variable necrosis, mainly observed in acute pulmonary histoplasmosis. The second shows well-formed, necrotising granulomas surrounded by a rim of epithelioid histiocytes and a fibrotic capsule. These lesions, also called "histoplasmomas", are often resected in the work-up of a solitary pulmonary nodule $[2,5]$. The third histological subtype is mostly observed in disseminated disease and consists of heavily infected histiocytes within the interstitium [2, 6]. $H$. capsulatum are usually not visible in granulomas on haematoxylin-eosin stains but GMS stains enlighten small, uniform, ovoid organisms, sometimes typically narrow-based [1, 4].

Treatment is only proven to be effective in severe pulmonary and disseminated forms. The agent of choice is amphotericin B for the most severe syndromes. It is recommended to use the liposomal form rather than the deoxycholate because of the lower nephrotoxicity. Itraconazole is used as a maintenance drug and in milder cases. Treatment can last from weeks to months, depending on the severity of the infection [1, 7].

In conclusion, this case highlights the importance of pathology and reminds us of the endemically important differential diagnoses for sarcoidosis. It also proves that, in all countries, the first step when analysing lung granulomas should be to rule out infection. 
A. Sibille*, P. Van Bleyenbergh*, K. Lagrou ${ }^{\#}$, A. Verstraeten and W. Wuyts*

Depts of *Pneumology, and "Microbiology, University Hospitals Leuven, Leuven, and 'Dept of Pneumology, AZ Sint-Lucas, Ghent, Belgium.

Correspondence: W. Wuyts, University Hospital of Leuven, Dept of Pneumology, Herestraat 49, 3000 Leuven, Belgium. Email: wim.wuyts@uzleuven.be

Statement of Interest: None declared.

\section{REFERENCES}

1 Kauffman CA. Histoplasmosis. Clin Chest Med 2009; 30: 217-225, v.
2 Mukhopadhyay S, Gal AA. Granulomatous lung disease: an approach to the differential diagnosis. Arch Pathol Lab Med 2010; 134: 667-690.

3 Kauffman CA. Histoplasmosis: a clinical and laboratory update. Clin Microbiol Rev 2007; 20: 115-132.

4 Wheat LJ. Improvements in diagnosis of histoplasmosis. Expert Opin Biol Ther 2006; 6: 1207-1221.

5 Puckett TF. Pulmonary histoplasmosis; a study of twenty-two cases with identification of $H$. capsulatum in resected lesions. Am Rev Tuberc 1953; 67: 453-476.

6 El-Zammar OA, Katzenstein AL. Pathological diagnosis of granulomatous lung disease: a review. Histopathology 2007; 50: 289-310.

7 Wheat LJ, Freifeld AG, Kleiman MB, et al. Clinical practice guidelines for the management of patients with histoplasmosis: 2007 update by the Infectious Diseases Society of America. Clin Infect Dis 2007; 45: 807-825.

\section{An unusual presentation of sarcoidosis with tetraplegia and severe osteolytic bone lesions}

\section{To the Editors:}

Sarcoidosis is a granulomatous multisystem disorder of unknown aetiology. Pathology is characterised by non-necrotising granulomas, which may affect virtually any organ, most commonly the lungs, lymph nodes, skin and eyes. Lytic foci in the phalanges of the hands and feet are common, whereas it is rare to see a widespread effect to the bone.

We present a case of severe diffuse osteolytic bone involvement and tetraplegia presenting as the first manifestation of sarcoidosis.

A 54-yr-old male was admitted to hospital after a bike accident, presenting with total paralysis of his left arm and paresis of his right arm and both legs. Computed tomography (CT) revealed fracture of C2 and widespread osteolytic lesions in the vertebrae, sternum, clavicles, skull, costae and pulmonal nodular infiltrates. The patient had no respiratory complaints.

Positron emission tomography with ${ }^{18}$ F-fluoro-2-deoxy-Dglucose (FDG-PET)/CT showed areas of pathological uptake that would suggest malignancy, but biopsy from the bone marrow showed non-necrotising granulomas compatible with sarcoidosis. The suspicion of cancer was maintained due to the rareness of osteolytic sarcoidosis, but repeated biopsies from os ileum and os parietale confirmed the diagnosis of sarcoidosis, and prednisolone and methotrexate were initiated. A stabilising operation of the cervical spine was performed; 2 months later, another operation with laminectomy at C1 level was necessary for decompression of the spinal cord.

At the time of diagnosis, pulmonary function tests showed a forced expiratory volume in $1 \mathrm{~s}$ (FEV1) of $1.8 \mathrm{~L}(43 \%$ of predicted), rising to $2.9 \mathrm{~L}$ (69\% pred) 6 months later. FEV1/ forced vital capacity (FVC) was normal; total lung capacity (74\% pred) and diffusion capacity (76\% pred) were both slightly decreased and did not improve with treatment.
Interleukin (IL)-2 receptor was elevated to $1,180 \mathrm{kU} \cdot \mathrm{L}^{-1}$ (normal range $223-710 \mathrm{kU} \cdot \mathrm{L}^{-1}$ ), whereas angiotensineconverting enzyme (ACE) was normal. There was a light hypercalcaemia $\left(1.43 \mathrm{mmol} \cdot \mathrm{L}^{-1}\right)$, slightly elevated C-reactive protein $\left(134 \mathrm{nmol} \cdot \mathrm{L}^{-1}\right)$ and a mild normochrome, normocytic anaemia $\left(7.5 \mathrm{mmol} \cdot \mathrm{L}^{-1}\right)$. These parameters were normalised after a few months of treatment.

Response to treatment was monitored with serum ACE, IL-2 receptor and bone scintigraphy. FDG-PET/CT and bone scintigraphy were performed early in the course of disease, and showed excellent correspondence (figs 1 and 2). Bone scintigraphy was chosen for monitoring.

Remission of the scintigraphic bone manifestations began after more than 1 yr of treatment, and was almost complete after 2 yrs. IL-2 receptor fell to normal levels within 2 months.

Prednisolone was gradually tapered and finally stopped after nearly 2 yrs of treatment. Immunosuppressive treatment was completed with intravenous biphosphonate (zoledronic acid), administered yearly for $3 \mathrm{yrs}$, and daily supplementation of calcium and vitamin D.

The patient underwent intensive rehabilitation after the operation with very satisfactory results. After 3 months, he was able to walk again, and he resumed work 2 yrs after the diagnosis. At the time of writing, the patient was still taking methotrexate and the disease was well controlled; methotrexate treatment was planned to be ceased during the coming months.

Bone involvement is frequent in sarcoidosis, and is seen in up to $13 \%$ of patients [1]. It is usually a late manifestation and is often associated with chronic pulmonary, cutaneous or multivisceral sarcoidosis. The most common manifestations are asymptomatic lytic lesions of the phalanges of the hands and feet [2]. Involvement of the vertebral column, the skull and the 